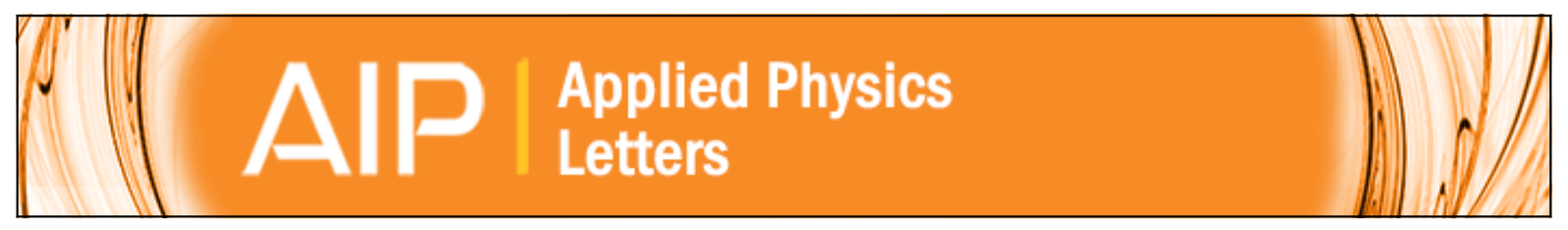

\title{
Fractal cluster modeling of the fatigue behavior of lead zirconate titanate
}

Shashank Priya, Hyeoung Woo Kim, Jungho Ryu, Kenji Uchino, and Dwight Viehland

Citation: Applied Physics Letters 80, 1625 (2002); doi: 10.1063/1.1455699

View online: http://dx.doi.org/10.1063/1.1455699

View Table of Contents: http://scitation.aip.org/content/aip/journal/apl/80/9?ver=pdfcov

Published by the AIP Publishing

Over 700 papers \&

presentations on

multiphysics simulation vew Now

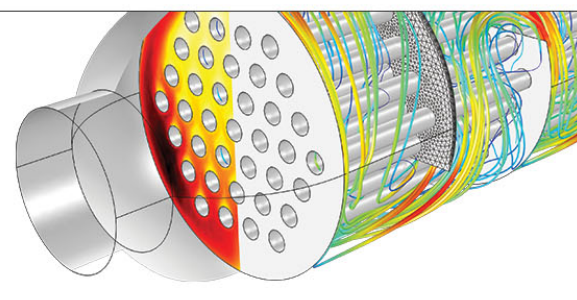




\title{
Fractal cluster modeling of the fatigue behavior of lead zirconate titanate
}

\author{
Shashank Priya, ${ }^{\text {a) }}$ Hyeoung Woo Kim, Jungho Ryu, and Kenji Uchino \\ International Center for Actuators and Transducers, Materials Research Institute, \\ Pennsylvania State University, University Park, Pennsylvania 16802 \\ Dwight Viehland \\ Department of Materials Science and Engineering, Virginia Tech, Blacksburg, Virginia 24061
}

(Received 3 October 2001; accepted for publication 20 December 2001)

\begin{abstract}
The fatigue behavior of lead zirconate titanate ceramics (PZT) has been studied under electrical and mechanical drives. Piezoelectric fatigue was studied using a mechanical method. Under ac mechanical drive, hard and soft PZTs showed an increase in the longitudinal piezoelectric constant at short times, reaching a maximum at intermediate times. Systematic investigations were performed to characterize the electrical fatigue behavior. A decrease in the magnitude of the remanent polarization was observed, which was relatively high for soft PZT. The dynamics of fatigue have been shown to scale to a hierarchical relaxation process, and this is typical of random field systems. (C) 2002 American Institute of Physics. [DOI: 10.1063/1.1455699]
\end{abstract}

In ferroelectrics, decay in remanent polarization $\left(P_{r}\right)$ under continuous bipolar excitation is classified as fatigue. The mechanisms responsible for fatigue in ferroelectric perovskites have been widely investigated. ${ }^{1-4}$ Fatigue occurs as a result of the ordering of oxygen vacancies in twodimensional (2D) planar arrays, resulting in the pinning of domain walls. ${ }^{5,6}$ Previous studies have shown that planar ordering of oxygen vacancies occurs in perovskites with an increase in defect density, ${ }^{7-11}$ with a minority of vacancies remaining as uncorrelated point defects. Reflection highenergy electron diffraction studies by Matsumoto et al. ${ }^{12}$ of $\mathrm{SrTiO}_{3}$ have shown that oxygen vacancies form a superstructure on a nanometer scale, further confirming the hypothesis that oxygen vacancies in pervoskites form an ordered network. With an increase in concentration, oxygen vacancies cluster, initially in one-dimensional (1D) chains and above a certain density in planes of specific geometries. Studies of fatigue anisotropy in relaxor-based ferroelectrics have shown that the activation energy for the domain migration is dependent on the crystallographic orientation and that the mobility is maximum along the $[001]_{c}$ direction. ${ }^{13}$ Thus, clustering of the polar entities occurs preferentially along well defined planes depending upon the symmetry of the parent phase. However, uncertainty remains as to how these oxygen vacancy complexes are distributed throughout the crystal.

Lead zirconate titanate solid solution $\left[\mathrm{Pb}\left(\mathrm{Zr}_{1-x} \mathrm{Ti}_{x}\right) \mathrm{O}_{3}\right.$ (PZT)] is a well known ferroelectric. Modified compositions of PZT are designated as "hard" if lower valent species are substituted onto the $A$ site, ${ }^{14}$ and "soft" if higher valent species are substituted onto the $A$ site. ${ }^{15}$ Hardening results in reduced dielectric and piezoelectric constants, lower dielectric losses, and higher coercive fields whereas softening results in increased dielectric and piezoelectric constants, reduced coercive fields, and enhanced dielectric losses. ${ }^{15}$ Hard PZTs are more stable under a continuous cyclical drive, compared to soft ones, due to their inherent polarization pinning

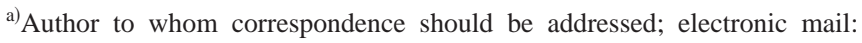
sup103@psu.edu
}

effects. However, previous investigations of soft PZT type materials under a continuous ac electrical drive have shown significant changes in domain configuration and strong fatigue in $P_{r}$ over time. ${ }^{6,16,17}$

Recent polarization investigations of soft PZTs have shown that polarization switching occurs by heterogeneous nucleation in the vicinity of local fields of randomly distributed point defects. ${ }^{18,19}$ Accordingly, polarization switching does not occur from the creation and motion of coherent micron-sized domains, but, rather, by the creation of polar clusters within the domains. These polar clusters that are induced under electric field are similar in size to those that exist in the field-cooled state of relaxor ferroelectrics. ${ }^{14}$ The results indicate that switching occurs through a relaxor-like condition in soft piezoelectrics.

Fatigue in relaxor systems has previously been shown to occur by a hierarchial mechanism, ${ }^{20}$ similar to in glassy states. ${ }^{21}$ This kind of fatigue process is intrinsically related to the search through configurational space for the lowest energy state. The macroscopic nonequilibrium fatigue characteristics under drive are governed by stretched exponential type functions. Stretched exponential type functions have previously been used to understand the unique dynamics of PMN-PT relaxor ferroelectrics under a weak field drive. ${ }^{22}$ The purpose of this study was to investigate the fatigue behavior of PZTs modified by ac mechanical and electrical cycling and to investigate the underlying mechanisms.

Hard and soft PZT ceramic specimens were fabricated by conventional mixed oxide methods and sintering procedures. The soft PZTs were made using PZT 5A powders obtained from Morgan Electroceramics Inc. (Columbus, $\mathrm{OH})$. The hard PZT samples were made using APC-841 powders obtained from American Piezoceramics Inc. (Mackeyville, PA). Plate-like specimens with an area of $44 \times 6 \mathrm{~mm}^{2}$ were pressed using cold isostatic pressing at pressure of 272 MPa for $10 \mathrm{~min}$ and then sintered at $1200^{\circ} \mathrm{C}$ for $2 \mathrm{~h}$ in a $\mathrm{PbO}$-atmosphere powder bed. After sintering, the plates were ground and machined to a thickness of either 1 or $0.5 \mathrm{~mm}$. Specimens of $5.5 \times 3.0 \mathrm{~mm}^{2}$ were used for the mechanical 
fatigue experiment, and specimens of $2.5 \times 2.5 \mathrm{~mm}^{2}$ were used for electrical fatigue experiments. All specimens were electroded by gold sputtering. All specimens were poled at $120^{\circ} \mathrm{C}$ under an electric field of $30 \mathrm{kV} / \mathrm{cm}$. Piezoelectric fatigue experiments were performed using a method whose experimental details were described in a previous letter. ${ }^{23}$ The stress $(\sigma)$ on the sample was computed from the initial magnitude of $d_{33}$ and charge ( $Q$ ) generated on the piezoelectric at the start of the experiment, $\sigma=d_{33} / Q A$ where $A$ is the area of the electroded surface. The maximum stress generated was $\sim 7 \mathrm{kPa}$. The ferroelectric properties were measured using a Radiant Technology RT66A system operating in virtual ground mode. Samples were immersed in Galden HT200 , an insulating liquid, to prevent arcing during the measurement process. The fatigue experiments consisted of continuously cycling the PZT samples using square pulses.

In disordered magnetic systems ${ }^{21,24}$ aging is known to be an inherent property. In these systems, many different metastable states which are nearly degenerate exist. Gaussiantype functions were used to analyze the relaxation time distributions [shown in Eq. (1)] in terms of $d_{33}$,

$$
d_{33}=d_{33}(0)+D_{r} \exp \left[-\left(\frac{t-t_{c}}{R_{t}}\right)^{n}\right]
$$

where $D_{r}$ is the distribution function at the initial time, $t_{c}$ is a characteristic relaxation time, $R_{t}$ is a displacement constant, and $d_{33}(0)$ and $n$ are constants. Different values of $n$ have been reported in the relaxation time distribution for various types of disordered magnetic systems. ${ }^{25}$ In randomfield systems, $n$ has been reported to be equal to 2, resulting in a normal Gaussian distribution whereas in glassy systems, $n$ is less than 2, resulting in a stretched or non-Gaussian distribution.

Equation (1) was used to fit the time dependence of the piezoelectric constant, which was measured under continuous drive. Data are shown in Figs. 1(a) and 1(b) for soft and hard PZTs, respectively. The fitting of the data to Eq. (1) is shown as a solid line in Fig. 1. The experimental results fit well with Eq. (1), with the restriction that the exponent equals 2. This demonstrates that the dynamics are Gaussian, consistent with that expected in a random-field system. Previous investigations of the dynamics of relaxor ferroelectric systems have revealed non-Gaussian dynamics in the dielectric relaxation spectra, ${ }^{25}$ which became increasingly nonGaussian with an increased reduction of the sample volume measured.

According to random-field theory, subdomains (or fractals) are created within normal domains under the application of reverse field. ${ }^{26,27}$ The time dependence of the polarization is given by

$$
P(t)=P_{0} \exp \left\{-a\left[\ln \left(\frac{t}{\tau}\right)\right]^{n}\right\}
$$

where $P_{0}$ is the total polarization, $a$ is a constant, $\tau$ is the characteristic relaxation time, and $n$ is the dimensionality. Systematic investigations of the time dependence of the polarization were performed on hard and soft PZTs under high amplitude electrical drive $(E=20 \mathrm{kV} / \mathrm{cm})$. Data are shown in Figs. 2(a) and 2(b) for hard and soft PZTs, respectively. The fit to Eq. (2) is shown as a solid line in Fig. 2. The fit
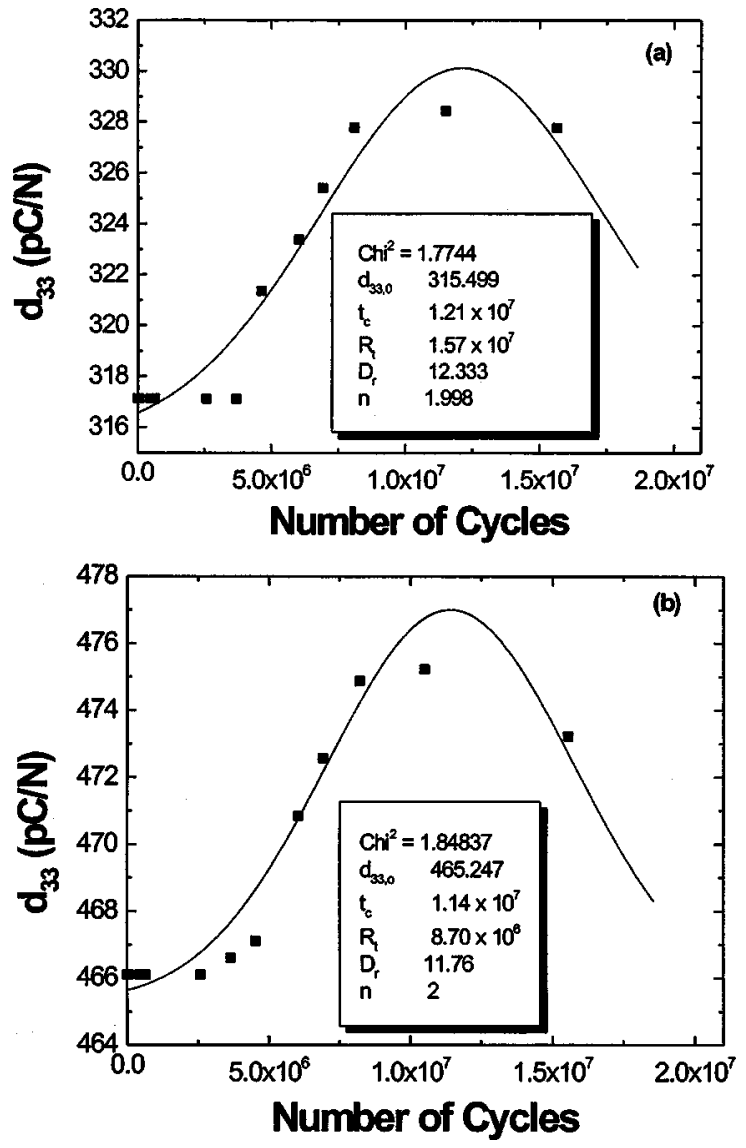

FIG. 1. Dependence of the longitudinal piezoelectric constant on the number of ac mechanical cycles for piezoceramics. (a) Hard PZT and (b) soft PZT.

parameters are also shown. Good correlation between the data and Eq. (2) can be seen in Figs. 2(a) and 2(b). These results are consistent with the interpretation of polarization switching by heterogeneous nucleation in the vicinity of random fields. ${ }^{18,19}$ Accordingly, switching occurs by the creation of fractal domains (i.e., nuclei or small clusters of reversed polarization under a poled condition). Interestingly, the main difference between the hard and soft PZTs was the characteristic relaxation time.

In Fig. 3, ferroelectric hysteresis loops are shown for the initial measurement and the last measurement taken after $10^{5}$ cycles of $20 \mathrm{kV} / \mathrm{cm}$. For hard PZT there was no significant
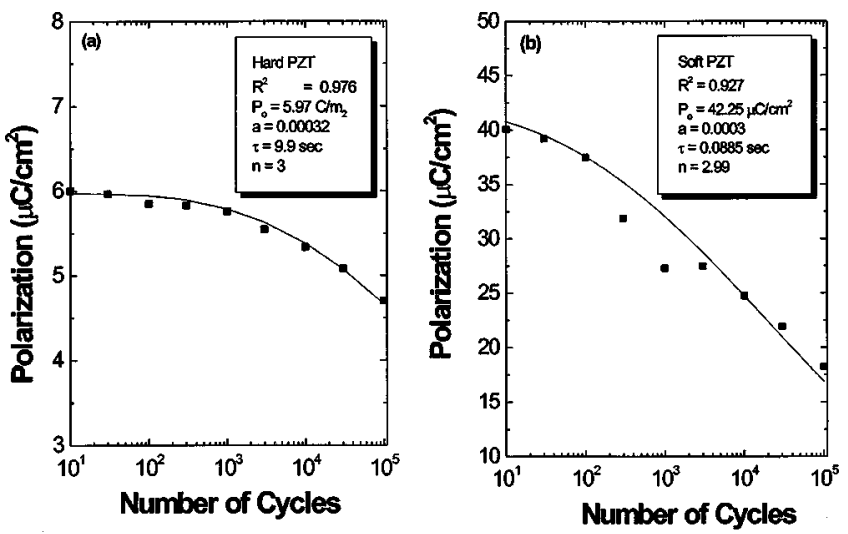

FIG. 2. Time dependence of the remanent polarization under high amplitude electrical drive $(E=20 \mathrm{kV} / \mathrm{cm})$ for (a) hard PZT and (b) soft PZT. 

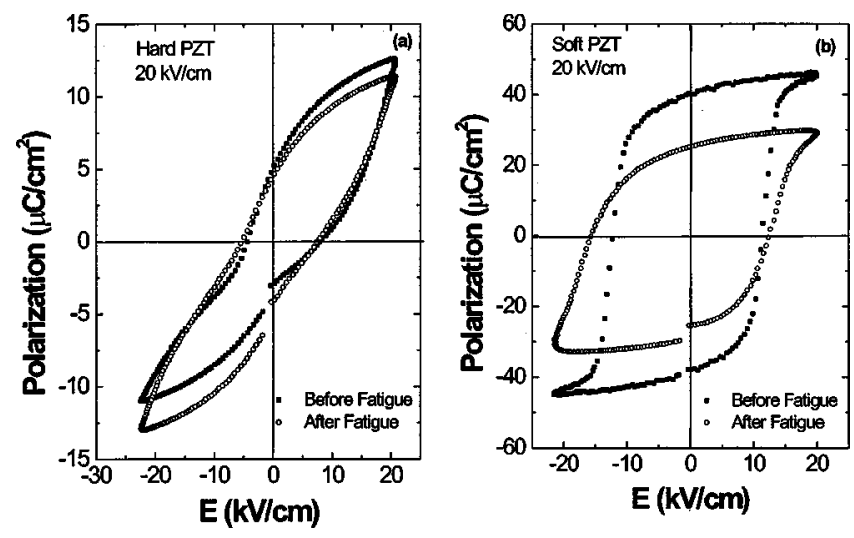

FIG. 3. $P-E$ hysteresis loop before and after the fatigue experiment for (a) hard PZT and (b) soft PZT.

effect of electrical cycling, as can be seen in Fig. 3(a). In this case, the maximum induced polarization was $\sim 12.5$ $\mu \mathrm{C} / \mathrm{cm}^{2}$, and double-loop-like characteristics were observed in the $P-E$ responses, similar to those previously reported for aged ferroelectric ceramics, ${ }^{6}$ and the decrease in remanent polarization in the time domain investigated was $\sim 20 \%$. However, for soft PZT, much more significant changes were observed in the $P-E$ loops between measurements taken during the initial and $10^{5}$ cycles. These results indicate commonalities between the time-dependent or fatigue behavior of soft and hard PZTs, with the principal difference being the rate of fatigue.

In summary, in this letter we reported the fatigue behavior of hard and soft PZT's under ac mechanical and electrical drives. The results obtained scale with the random-field model. In this case, fractal domains of reverse polarization were gradually created in the infinite domain under excitation during switching.

This research was sponsored by the Office of Naval Research through Contract No. N00014-99-J-0754.
${ }^{1}$ H. L. Stadler, J. Appl. Phys. 29, 743 (1958).

${ }^{2}$ S. D. Bernstein, J. Y. Wang, Y. Kisler, and R. W. Tustison, J. Mater. Res. 8, 12 (1992).

${ }^{3}$ W. L. Warren, D. Dimos, B. A. Tuttle, C. E. Pike, R. W. Schwartz, P. L. Claws, and P. C. McIntyre, J. Appl. Phys. 77, 6695 (1995).

${ }^{4}$ A. Gruverman, O. Auciello, and H. Tokumoto, Appl. Phys. Lett. 69, 3191 (1996).

${ }^{5}$ J. F. Scott and M. Dawber, Appl. Phys. Lett. 76, 3801 (2000).

${ }^{6}$ Q. Tan, Ph.D. dissertation, University of Illinois, Urbana, IL, 1998.

${ }^{7}$ J. F. Scott, C. A. Araujo, B. M. Melnick, L. D. McMillan, and R. Zuleeeg, J. Appl. Phys. 70, 382 (1991).

${ }^{8}$ G. Arlt and H. Neumann, Ferroelectrics 87, 109 (1988).

${ }^{9}$ C. Brennan, Integr. Ferroelectr. 8, 93 (1995).

${ }^{10}$ I. K. Yoo and S. B. Desu, Phys. Status Solidi A 133, 565 (1992).

${ }^{11}$ A. Yu Kudzin, T. V. Panchenko, and S. P. Yudin, Fiz. Tverd. Tela (S.Petersburg) 16, 1589 (1975).

${ }^{12}$ T. Matsumoto, H. Tanaka, K. Kouguchi, T. Kawai, and S. Kawai, Surf. Sci. 312, 21 (1994).

${ }^{13}$ K. Takemura, M. Ozgul, V. Bornand, S. T. McKinstry, and C. Randall, J. Appl. Phys. 88, 7272 (2000).

${ }^{14}$ X. H. Dai, Z. Xu, and D. Viehland, Philos. Mag. B 70, 33 (1994).

${ }^{15}$ B. Jaffe, W. Cook, and H. Jaffe, in Piezoelectric Ceramics, edited by J. P. Roberts and P. Popper (Academic, London, 1971).

${ }^{16}$ V. E. Borodin, E. I. Eknadiosyants, and A. P. Pinskaya, Semiconductor Ferroelectrics (Kniga, Rostov, 1996), Vol. 6, p. 125.

${ }^{17}$ E. L. Colla, S. Hong, D. V. Taylor, A. K. Taganstev, N. Setter, and K. No, Appl. Phys. Lett. 72, 2763 (1998).

${ }^{18}$ D. Viehland and Y. H. Chen, J. Appl. Phys. 88, 6696 (2000).

${ }^{19}$ D. Viehland and J. F. Li, J. Appl. Phys. 90, 2995 (2001).

${ }^{20}$ E. Colla, L. Chao, M. Weissman, and D. Viehland, Phys. Rev. Lett. 85, 14 (2000).

${ }^{21}$ J. Mydosh, Spin Glasses, An Experimental Introduction (Taylor and Francis, London, 1993).

${ }^{22}$ D. Viehland, M. Wuttig, and L. E. Cross, Ferroelectrics 120, 71 (1991).

${ }^{23}$ S. Priya, J. Ryu, K. Uchino, and D. Viehland, Appl. Phys. Lett. 79, 2624 (2001).

${ }^{24}$ S. L. Hutton, U. T. Höchli, and M. Magilone, Relaxation in Complex Systems and Related Topics, edited by I. A. Campbell and C. Giovannella (Plenum, New York, 1990), p. 289.

${ }^{25}$ K. P. O'Brien, M. B. Weissman, D. Sheehy, and D. Viehland, Phys. Rev. B 56, 11365 (1997).

${ }^{26}$ W. Kleemann, Int. J. Mod. Phys. 7, 2469 (1993).

${ }^{27}$ Y. Imry and S. Ma, Phys. Rev. Lett. 35, 1399 (1975). 